\title{
Comparison of the best approximation of holomorphic func- tions from Hardy space
}

\author{
F. G. Abdullayeve, ${ }^{\mathrm{a}, \mathrm{b}}$, V. V. Savchuk ${ }^{\mathrm{c}, *}$, D. Şimşek ${ }^{\mathrm{b}}$ \\ ${ }^{a}$ Mersin University, Mersin, Turkey. \\ ${ }^{b}$ Kyrgyz-Turkish Manas University, Bishkek, Kyrgyzstan. \\ ${ }^{c}$ Institute of Mathematics of NAS of Ukraine, Kyiv, Ukraine.
}

\begin{abstract}
We compare the best approximations of holomorphic functions in the Hardy space $\mathrm{H}^{1}$ by algebraic polynomials and trigonometric polynomials. Particulary, we establish a class of functions $f \in \mathrm{H}^{1}$ for which the best trigonometric approximation do not coincide with the best algebraic approximation.
\end{abstract}

Keywords: Best approximation, Hardy space, non-negative trigonometric polynomials.

2010 MSC: 30C45, 30C50.

(C)2019 All rights reserved.

\section{Introduction and main results}

Let $\mathbb{D}:=\{z \in \mathbb{C}:|z|<1\}, \mathbb{T}:=\{z \in \mathbb{C}:|z|=1\}$ and let $\mathrm{dm}$ be a normalized Lebesgue measure on $\mathbb{T}$. The Hardy space $\mathrm{H}^{\mathrm{q}}$ for $1 \leqslant \mathrm{q} \leqslant \infty$ is the class of holomorphic in the $\mathbb{D}$ functions $\mathrm{f}$ satisfied $\|\mathrm{f}\|_{\mathrm{q}}<\infty$, where

$$
\|f\|_{\mathrm{q}}:= \begin{cases}\sup _{\rho \in(0,1)}\left(\int_{\mathbb{T}}|\mathrm{f}(\rho \mathrm{t})|^{\mathrm{q}} \mathrm{dm}(\mathrm{t})\right)^{1 / \mathrm{q}}, & 1 \leqslant \mathrm{q}<\infty, \\ \sup _{z \in \mathbb{D}}|\mathrm{f}(z)|, & \mathrm{q}=\infty .\end{cases}
$$

It is well-known that each function $f$ from $H^{q}$ has the nontangential limits $f(t), t \in \mathbb{T}$, almost everywhere and

$$
\|f\|_{q}= \begin{cases}\left(\int_{\mathbb{T}}|f|^{q} d m\right)^{1 / q}, & 1 \leqslant q<\infty, \\ \underset{t \in \mathbb{T}}{\operatorname{ess} \sup |f(t)|,} & q=\infty .\end{cases}
$$

\footnotetext{
*Corresponding author

Email addresses: fabdul@mersin.edu.tr (F. G. Abdullayev), savchuk@imath.kiev.ua (V. V. Savchuk), dagistan.simsek@manas.edu.kg (D. Şimşek)
}

doi: $10.22436 /$ jnsa.012.07.01

Received: 2018-10-22 Revised: 2019-01-18 Accepted: 2019-01-25 
The best polynomial approximation of $f \in H^{q}$ of order $n, n \in \mathbb{N}$, is the quantity

$$
E_{n}(f)_{q}:=\inf _{P \in \mathcal{P}_{n-1}}\|f-P\|_{q}, \quad n \in \mathbb{N}
$$

where $\mathcal{P}_{n-1}$ is the set of all algebraic polynomials of degree at most $n-1$.

We will denote by $\mathcal{T}_{n-1}$ the set of all trigonometric polynomials of degree at most $n-1$ on the circle $\mathbb{T}$ with complex coefficients, that is a functions of the form $T(t)=\sum_{|k| \leqslant n-1} c_{k} t^{k}, t \in \mathbb{T}$.

The best trigonometric approximation of $f \in \mathrm{H}^{\mathrm{q}}$ of order $n, n \in \mathbb{N}$, is the quantity

$$
\widetilde{E}_{\mathfrak{n}}(f)_{\mathrm{q}}:=\inf _{\mathrm{T} \in \mathcal{T}_{n-1}}\|f-\mathrm{T}\|_{\mathrm{q}}, \quad \mathrm{n} \in \mathbb{N} \text {. }
$$

The polynomials $P^{*}$ and $T^{*}$ satisfied $\left\|f-P^{*}\right\|_{q}=E_{n}(f)_{q}$ and $\left\|f-T^{*}\right\|_{q}=\widetilde{E}_{n}(f)_{q}$ are called a best approximation to $f$ among the set $\mathcal{P}_{n-1}$ and $\mathcal{T}_{n-1}$ respectively in the metric $\|\cdot\|_{q}$.

Obviously, for any $1 \leqslant \mathrm{q} \leqslant \infty$, one has

$$
\widetilde{E}_{1}(f)_{q}=E_{1}(f)_{q},
$$

and

$$
\widetilde{\mathrm{E}}_{\mathrm{n}}(\mathrm{f})_{\mathrm{q}} \leqslant \mathrm{E}_{\mathrm{n}}(\mathrm{f})_{\mathrm{q}}, \quad \forall \mathrm{n} \in \mathbb{N} \backslash\{1\} .
$$

Pekarskii [3] was the first to point out in print that there exists a function $f \in \mathrm{H}^{\infty}$ such that

$$
\widetilde{\mathrm{E}}_{n}(f)_{\infty}<\mathrm{E}_{n}(f)_{\infty},
$$

for a given natural $n>1$.

Particularly, in [3] it was shown that for the sequence $\left\{f_{n, \rho}\right\}_{0<\rho<1}$ of functions

$$
f_{n, \rho}(z)=z^{n} \frac{1-\rho^{2(n+1)}}{1-\rho^{n+1} z^{n+1}}, \quad n \in \mathbb{N} \backslash\{1\},
$$

we have

$$
\widetilde{E}_{n}\left(f_{n, \rho}\right)_{\infty}=1<E_{n}\left(f_{n, \rho}\right)_{\infty}=\left\|f_{n, \rho}\right\|_{\infty}=1+\rho^{n+1} \rightarrow 2 \text { as } \rho \rightarrow 1 .
$$

In view of these results, it is natural to assume that function $f_{n, \rho}$ must satisfy the inequality

$$
\widetilde{E}_{n}(f)_{1}<E_{n}(f)_{1} .
$$

As we will show later (see Proposition 2.4), this is indeed the case, but the method of [3] cannot be applied to proving this one.

Finally, let us pay attention that Pekarskii's example says nothing about the inequality (1.2) for an individual function for each natural $n$.

The aim of this note is to establish a class of functions $f$ satisfied (1.4) for a given $n$ as well as to construct an individual function $f$ for which (1.4) holds true for each natural $n$.

Let $\Pi_{n}, n \in \mathbb{N} \backslash\{1\}$, denote the set of all algebraic polynomials $f(z)=\sum_{k=0}^{3 n-1} a_{k} z^{k}$ of degree at most $3 n-1$ with complex coefficients satisfied $\left|a_{n}\right| \sum_{k=2 n+1}^{3 n-1}\left|a_{k}\right|>0$ and such that

$$
\min _{z \in \mathbb{T}} \operatorname{Re} \sum_{k=0}^{2 n-1} \frac{a_{n+k}}{a_{n}} z^{k} \geqslant \frac{1}{2} .
$$

Let $\widehat{f}_{k}=\frac{f^{(k)}(0)}{k !}, k=0,1, \cdots$, denote the Taylor coefficients of $f \in H^{1}$.

Our main results are the following two theorems. 
Theorem 1.1. Suppose $\mathrm{n} \in \mathbb{N} \backslash\{1\}$ and $\mathrm{f} \in \Pi_{\mathrm{n}}$. Then

$$
\left|\widehat{f}_{n}\right|=\widetilde{E}_{n}(f)_{1}<E_{n}(f)_{1} .
$$

Theorem 1.2. Suppose that $0<\rho<1$ and let $f(z)=1 /(1-\rho z)$. Then for each natural $n$,

$$
\widetilde{\mathrm{E}}_{n}(f)_{1}=\frac{2}{\pi} \rho^{n} \mathbf{K}\left(\rho^{2 n}\right),
$$

and

$$
E_{n}(f)_{1}=\frac{2}{\pi} \rho^{n} K\left(\rho^{n+1}\right),
$$

where

$$
\mathbf{K}(x)=\int_{0}^{\frac{\pi}{2}} \frac{\mathrm{d} \theta}{\sqrt{1-x^{2} \sin ^{2} \theta}},
$$

is the complete elliptic integral of the first kind.

Corollary 1.3. Suppose that $0<\rho<1$ and let $\mathrm{f}(z)=1 /(1-\rho z)$. Then for each natural $\mathrm{n}>1$ the inequality (1.2) holds true.

\section{Examples}

In this section, our goal is to prove the following propositions that may be considered as examples to the main theorems.

Firstly, we give some examples of polynomials from $\Pi_{n}$.

Proposition 2.1. Let $n, p \in \mathbb{N}, n \leqslant p \leqslant 2 n-1$ and let $A_{p}(t)=\sum_{|k| \leqslant p} a_{k} t^{k}, B_{2 n-p-1}(t)=\sum_{|k| \leqslant 2 n-p-1} b_{k} t^{k}$, $\left|a_{\mathfrak{p}} b_{2 n-p-2}\right|>0$ be two trigonometric polynomials, such that $A_{p}(t) B_{2 n-p-1}(t) \geqslant 0$ for all $t \in \mathbb{T}$. Then the function

$$
f(z)=z^{n}\left(\sum_{k=0}^{p} a_{k} z^{k}\right)\left(\sum_{|k| \leqslant 2 n-p-1} b_{k} z^{k}\right),
$$

belongs to $\Pi_{\mathrm{n}}$ and

$$
\widetilde{\mathrm{E}}_{\mathrm{n}}(\mathrm{f})_{1}=\widehat{\mathrm{f}}_{\mathrm{n}}=\mathrm{a}_{0} \mathrm{~b}_{0}+2 \operatorname{Re} \sum_{\mathrm{k}=1}^{2 \mathrm{n}-\mathrm{p}-1} \mathrm{a}_{\mathrm{k}} \overline{\mathrm{b}}_{\mathrm{k}}
$$

where for $\mathrm{p}=2 \mathrm{n}-1, \sum_{k=1}^{0}=0$. Moreover, the trigonometric polynomial

$$
T(t)=-t^{n}\left(\sum_{k=1}^{p} a_{-k} t^{-k}\right) B_{2 n-p-1}(t)
$$

is the best approximation to f among the set $\mathcal{T}_{\mathrm{n}-1}$ in the metric $\|\cdot\|_{1}$.

Indeed, a straightforward calculation shows that $T$ has the form $T(t)=\sum_{k=-(n-1)}^{3 n-2 p-1} c_{k} t^{k}, c_{k} \in \mathbb{C}$. Therefore, $T \in \mathcal{T}_{n-1}$, since $3 n-2 p-1 \leqslant n-1$.

As it is easy to see that

$$
t^{-n}(f(t)-T(t))=A_{p}(t) B_{2 n-p-1}(t) \geqslant 0, \quad t \in \mathbb{T},
$$

then

$$
\widetilde{\mathrm{E}}_{n}(f)_{1} \leqslant\|f-T\|_{1}=\int_{\mathbb{T}} A_{p} B_{2 n-p-1} d m=a_{0} b_{0}+2 \operatorname{Re} \sum_{k=1}^{2 n-p-1} a_{k} \bar{b}_{k} .
$$


Since $\left|\widehat{\mathrm{f}}_{\mathrm{n}}\right| \leqslant \widetilde{\mathrm{E}}_{\mathrm{n}}(\mathrm{f})_{1}$ and

$$
\widehat{f}_{n}=\int_{\mathbb{T}} f(t) t^{-n} d m(t)=\int_{\mathbb{T}}(f(t)-T(t)) t^{-n} d m(t)=\int_{\mathbb{T}} A_{p} B_{2 n-p-1} d m \geqslant \widetilde{E}_{n}(f)_{1},
$$

we get $\widetilde{E}_{n}(f)_{1}=\widehat{f}_{n}$. As it follows from Lemma 3.1 below,

$$
\widetilde{\mathrm{E}}_{\mathrm{n}}(\mathrm{f})_{1}=\left|\widehat{\mathrm{f}}_{\mathrm{n}}\right| \Longleftrightarrow \min _{\mathrm{t} \in \mathbb{T}} \sum_{k=0}^{2 \mathrm{n}-1} \frac{\widehat{\mathrm{f}}_{\mathrm{k}+\mathrm{n}}}{\widehat{\mathrm{f}}_{\mathrm{n}}} \mathrm{t}^{\mathrm{k}} \geqslant \frac{1}{2} .
$$

Therefore $f \in \Pi_{n}$, because $\left|\widehat{f}_{n}\right| \sum_{k=n+1}^{3 n-1}\left|\widehat{f}_{k}\right| \geqslant\left|\widehat{f}_{n} \widehat{f}_{3 n-1}\right|=\left|\widehat{f}_{n} a_{p} b_{2 n-p-1}\right|>0$.

The computation of the value of best approximation $E_{n}(f)_{1}$ for function from $\Pi_{n}$ is a more complicated problem. In the next two propositions we would like to pay attention to how a sieve-method may be applied to computation of this one.

Let $r \in \mathbb{Z}_{+}$and $s \in \mathbb{N}$. The linear operator $\mathcal{W}_{r, s}$ defined on $\mathrm{H}^{1}$ by

$$
\mathcal{W}_{r, s}(f)(z)=\sum_{j=0}^{\infty} \widehat{f}_{j s+r} z^{j}, \quad z \in \mathbb{D},
$$

is called the sieve operator.

The importance of sieve operator for the theory of approximation of holomorphic functions is recognized by the following.

Proposition 2.2. Suppose that $1 \leqslant q \leqslant \infty, r \in \mathbb{Z}_{+}, s \in \mathbb{N}, s \geqslant r+1$ and let

$$
\mathcal{G}_{\mathrm{r}, \mathrm{s}}^{\mathrm{q}}:=\left\{\mathrm{g} \in \mathrm{H}^{\mathrm{q}}: \mathcal{W}_{\mathrm{r}, \mathrm{s}}(\mathrm{g})=0\right\} \text {. }
$$

Then for any function $\mathrm{f} \in \mathrm{H}^{p}$,

$$
\begin{aligned}
\left\|\mathcal{W}_{r, s}(f)\right\|_{q} & =\min _{g \in \mathcal{G}_{r, s}^{q}}\|f-g\|_{q} \\
& \leqslant E_{r}(f)_{q} .
\end{aligned}
$$

The minimum in (2.2) is attained for the function $g(z)=f(z)-z^{r} \mathcal{W}_{r, s}(f)\left(z^{s}\right)$. The equality sign in (2.3) is attained for function of the form $f(z)=\sum_{j=0}^{\infty} a_{j} z^{j+r}$.

Proof. Indeed, for any $g \in \mathcal{G}_{r, s}^{\mathrm{q}}$,

$$
\mathcal{W}_{r, s}(f)=\mathcal{W}_{r, s}(f-g)
$$

Therefore,

$$
\left\|\mathcal{W}_{r, s}(f)\right\|_{q} \leqslant\left\|\mathcal{W}_{r, s}\right\|_{q}\|f-g\|_{q},
$$

where $\left\|\mathcal{W}_{r, s}\right\|_{q}$ is the norm of operator $\mathcal{W}_{r, s}$ on $\mathrm{H}^{q}$ space.

It was shown in [5] that

$$
\left\|\mathcal{W}_{r, s}\right\|_{\infty}=1 \Leftrightarrow s \geqslant r+1 \Rightarrow\left\|\mathcal{W}_{r, s}\right\|_{\mathbf{q}}=1 .
$$

Thus, for any $g \in \mathcal{G}_{r, s}^{q}$,

$$
\left\|\mathcal{W}_{r, s}(f)\right\|_{q} \leqslant\|f-g\|_{q} .
$$

It is clear that the best we can minimize the right side in the above inequality is to choose

$$
g(z)=f(z)-z^{r} \mathcal{W}_{r, s}(f)\left(z^{s}\right) .
$$

For proving (2.3) it suffices to note that $\mathcal{P}_{r-1} \subset \mathcal{G}_{r, s}^{q}$. 
Proposition 2.3. Suppose that $\mathrm{n} \in \mathbb{N} \backslash\{1\}$ and let $\mathrm{f}(z)=z^{\mathrm{n}}+\frac{1}{2} z^{2 \mathrm{n}+1}$. Then $\mathrm{f} \in \Pi_{\mathrm{n}}$,

$$
\widetilde{\mathrm{E}}_{\mathfrak{n}}(\mathrm{f})_{1}=1 \text {, }
$$

and

$$
E_{n}(f)_{1}=\|f\|_{1}=\frac{3}{\pi} \mathbf{E}\left(\frac{2 \sqrt{2}}{3}\right)
$$

where

$$
\mathbf{E}(x)=\int_{0}^{\frac{\pi}{2}} \sqrt{1-x^{2} \sin ^{2} \theta} \mathrm{d} \theta
$$

is a complete elliptic integral of the second kind.

Clearly, $f$ has the form (2.1), in which $p=n+1, a_{0}=b_{0}=1, a_{n+1}=\frac{1}{2}$, and $a_{k}=a_{n-1}=b_{k}=0$ for $k=1,2, \cdots, n-2$. Therefore, by Proposition 2.1, $f \in \Pi_{n}$ and $\widetilde{E}_{n}(f)_{1}=1$.

On the other hand, $\mathcal{W}_{n, n+1}(f)(z)=1+\frac{1}{2} z$. Thus, by Proposition 2.2

$$
\begin{aligned}
\mathrm{E}_{\mathrm{n}}(\mathrm{f})_{1} & =\|\mathrm{f}\|_{1} \\
& =\int_{\mathbb{T}}\left|1+\frac{\mathrm{t}}{2}\right| \mathrm{dm}(\mathrm{t}) \\
& =\frac{3}{4 \pi} \int_{-\pi}^{\pi} \sqrt{1-\frac{8}{9} \sin ^{2} \frac{\theta}{2}} \mathrm{~d} \theta \\
& =\frac{3}{\pi} \mathrm{E}\left(\frac{2 \sqrt{2}}{3}\right) \approx 1.01925 .
\end{aligned}
$$

Proposition 2.4. Suppose that $\mathrm{n} \in \mathbb{N} \backslash\{1\}, 0<\rho<1$ and let $\mathrm{f}_{\mathrm{n}, \rho}$ be the function defined in (1.3). Then

$$
\widetilde{\mathrm{E}}_{\mathrm{n}}\left(\mathrm{f}_{\mathrm{n}, \mathrm{\rho}}\right)_{1} \leqslant 1 \text {, }
$$

and

$$
E_{n}\left(f_{n, \rho}\right)_{1}=\left\|f_{n, \rho}\right\|_{1}=\frac{2}{\pi}\left(1-\rho^{2(n+1)}\right) \mathbf{K}\left(\rho^{n+1}\right) .
$$

Therefore, for the $\rho_{*} \approx(0.139793)^{\frac{1}{n+1}}$ that maximizes the function $\rho \mapsto\left(1-\rho^{2(n+1)}\right) \mathbf{K}\left(\rho^{n+1}\right)$ on $[0,1]$, we get (1.4).

Indeed,

$$
\widetilde{E}_{n}\left(f_{n, \rho}\right)_{1} \leqslant \widetilde{E}_{n}\left(f_{n, \rho}\right)_{\infty}=1
$$

On the other hand,

$$
z^{n} \mathcal{W}_{n, n+1}\left(f_{n, \rho}\right)\left(z^{n+1}\right)=f_{n, \rho}(z)
$$

Therefore, $E_{n}\left(f_{n, \rho}\right)_{1}=\left\|f_{n, \rho}\right\|_{1}$.

\section{Auxiliary lemmas}

The proof of Theorem 1.1 is based on the following assertions that are also of some independent interest.

Lemma 3.1. Suppose that $\mathrm{n} \in \mathbb{N}, \mathrm{f} \in \mathrm{H}^{1}$ and $\left|\widehat{\mathrm{f}}_{\mathrm{n}}\right|>0$. Then equality $\widetilde{\mathrm{E}}_{\mathrm{n}}(\mathrm{f})_{1}=\left|\widehat{\mathrm{f}}_{\mathrm{n}}\right|$ holds true if and only if

$$
f(z)=\sum_{k=0}^{3 n-1} \widehat{f}_{k} z^{k}, \quad\left|\widehat{f}_{n}\right|>0,
$$


and

$$
\min _{t \in \mathbb{T}} \operatorname{Re} \sum_{k=0}^{2 n-1} \frac{\widehat{f}_{n+k}}{\widehat{f}_{n}} t^{k} \geqslant \frac{1}{2}
$$

Moreover, the trigonometric polynomial

$$
T^{*}(t)=\sum_{k=0}^{n-1}\left(\widehat{f}_{k}-\overline{\widehat{f}_{2 n-k}} e^{i 2 \arg \widehat{f}_{n}}\right) t^{k}-e^{i 2 \arg \widehat{f}_{n}} \sum_{k=1}^{n-1} \overline{\hat{f}_{k+2 n+1}} t^{-k},
$$

is the unique best approximation to f among the set $\mathcal{T}_{\mathfrak{n}-1}$ in the metric $\|\cdot\|_{1}$.

Proof. By definition

$$
\begin{aligned}
\widetilde{\mathrm{E}}_{n}(f)_{1} & =\inf _{a_{k} \in \mathbb{C}} \int_{\mathbb{T}}\left|f(t)-\sum_{|k| \leqslant n-1} a_{k} t^{k}\right| d m(t) \\
& =\inf _{a_{k} \in \mathbb{C}} \int_{\mathbb{T}}\left|t^{n-1} f(t)-\sum_{k=0}^{2(n-1)} a_{k-n+1} t^{k}\right| d m(t)=E_{2 n-1}(g)_{1},
\end{aligned}
$$

where $g(t)=t^{n-1} f(t)$.

Therefore, taking into account that $\widehat{f}_{n}=\widehat{g}_{2 n-1}$, we get the equivalence

$$
\widetilde{\mathrm{E}}_{\mathrm{n}}(\mathrm{f})_{1}=\left|\widehat{\mathrm{f}}_{\mathrm{n}}\right| \Longleftrightarrow \mathrm{E}_{2 \mathrm{n}-1}(\mathrm{~g})_{1}=\left|\widehat{\mathrm{g}}_{2 \mathrm{n}-1}\right|
$$

In addition, the trigonometric polynomial $\sum_{|k| \leqslant n-1} a_{k} t^{k}$ is the best approximation to $f$ among the set $\mathcal{T}_{\mathfrak{n}-1}$ if and only if the algebraic polynomial $\sum_{k=0}^{2(n-1)} a_{k-n+1} t^{k}$ is the best approximation to $g$ among the set $\mathcal{P}_{2(n-1)}$.

The remainder of this proof follows immediately from the next lemma proved in [1].

Lemma 3.2. Suppose that $\mathrm{n} \in \mathbb{N}, \mathrm{f} \in \mathrm{H}^{1}$ and $\left|\widehat{\mathrm{f}}_{\mathrm{n}}\right|>0$. The equality $\mathrm{E}_{\mathrm{n}}(\mathrm{f})_{1}=\left|\widehat{\mathrm{f}}_{\mathrm{n}}\right|$ holds true if and only if

$$
f(z)=\sum_{k=0}^{2 n} \widehat{f}_{k} z^{k}
$$

and

$$
\min _{t \in \mathbb{T}} \operatorname{Re} \sum_{k=0}^{n} \frac{\widehat{f}_{n+k}}{\widehat{f}_{n}} t^{k} \geqslant \frac{1}{2}
$$

Moreover, the polynomial

$$
P^{*}(z)=\sum_{k=0}^{n-1}\left(\widehat{f}_{k}-\overline{\widehat{f}_{2 n-k}} e^{i 2 \arg \widehat{f}_{n}}\right) z^{k},
$$

is the unique best approximation to f among the set $\mathcal{P}_{\mathrm{n}-1}$ in the metric $\|\cdot\|_{1}$.

Lemma 3.3. Suppose that $0 \leqslant \rho<1$ and $p \in \mathbb{N}$. Then

$$
\int_{\mathbb{T}} \frac{\left|1-\rho^{p} t^{p}\right|}{|1-\rho t|^{2}} d m(t)=\frac{2}{\pi} \frac{1-\rho^{2 p}}{1-\rho^{2}} \mathbf{K}\left(\rho^{p}\right),
$$

where $\mathbf{K}$ is the complete elliptic integral of the first kind. 
This assertion essentially is contained in [6]. For convenience, we present here its proof.

Proof. From the expansion

$$
\frac{1}{\sqrt{1-x}}=1+\sum_{k=1}^{\infty} \alpha_{k} x^{k}, \quad \forall x \in \mathbb{D},
$$

where

$$
\alpha_{k}:=\frac{(2 k-1) ! !}{(2 k) ! !}, \quad k \in \mathbb{N}
$$

we get

$$
\begin{aligned}
\frac{\sqrt{1-\chi^{p}}}{1-\chi} & =\frac{1-\chi^{p}}{1-\chi} \frac{1}{\sqrt{1-\chi^{p}}} \\
& =\frac{1-\chi^{p}}{1-\chi}\left(1+\sum_{k=1}^{\infty} \alpha_{k} x^{p k}\right) \\
& =\frac{1-\chi^{p}}{1-\chi}+\sum_{k=1}^{\infty} \alpha_{k} \frac{1-\chi^{p}}{1-x} x^{p k} \\
& =\sum_{v=0}^{p-1} x^{v}+\sum_{k=1}^{\infty} \alpha_{k} \sum_{v=p k}^{p(k+1)-1} x^{v}, \quad \forall x \in \mathbb{D} .
\end{aligned}
$$

Therefore, by Parseval's identity,

$$
\begin{aligned}
\int_{\mathbb{T}} \frac{\mid 1-\rho^{p_{t}}{ }^{p}}{|1-\rho t|^{2}} \mathrm{dm}(\mathrm{t}) & =\frac{1}{2 \pi} \int_{0}^{2 \pi} \frac{\sqrt{1-\rho^{p} e^{i p \theta}}}{1-\rho e^{i \theta}} \frac{\sqrt{1-\rho^{p} e^{-i p \theta}}}{1-\rho e^{-i \theta}} \mathrm{d} \theta \\
& =\sum_{v=0}^{p-1} \rho^{2 v}+\sum_{k=1}^{\infty} \alpha_{k}^{2} \sum_{v=p k}^{p(k+1)-1} \rho^{2 v} \\
& =\frac{1-\rho^{2 p}}{1-\rho^{2}}\left(1+\sum_{k=1}^{\infty} \alpha_{k}^{2} \rho^{2 p k}\right) \\
& =\frac{1-\rho^{2 p}}{1-\rho^{2}} \frac{1}{2 \pi} \int_{0}^{2 \pi} \frac{1}{\left|1-\rho^{p} e^{-i \theta}\right|} d \theta \\
& =\frac{2}{\pi} \frac{1-\rho^{2 p}}{1-\rho^{2}} \mathbf{K}\left(\rho^{p}\right) .
\end{aligned}
$$

\section{Proofs of main results}

Proof of Theorem 1.1. It is ease to see that functions from the set $\Pi_{n}$ satisfies conditions of Lemma 3.1. Therefore, $\left|\widehat{f}_{n}\right|=\widetilde{E}_{n}(f)_{1}$. On the other hand, any function from $\Pi_{n}$ does not satisfy conditions of Lemma 3.2. Thus, for any $f \in \Pi_{n}, E_{n}(f)_{1} \neq\left|\widehat{f}_{n}\right|$. The result follows, since (1.1).

Proof of Theorem 1.2. First for all we note that as was shown by Alper [2] (see also some generalizations in [4])

$$
E_{p}(f)_{1}=\rho^{p} \frac{1-\rho^{2}}{1-\rho^{2(p+1)}} \int_{\mathbb{T}} \frac{\left|1-\rho^{p+1} t^{p+1}\right|}{|1-\rho t|^{2}} d m(t) .
$$

From this formula, by Lemma 3.3 we get the equality (1.6). 
Let us prove (1.5).

We have

$$
\begin{aligned}
& \widetilde{\mathrm{E}}_{\mathrm{n}}(f)_{1}=\min _{\mathrm{a}_{\mathrm{k}} \in \mathbb{C}} \int_{\mathbb{T}}\left|\frac{1}{1-\rho t}-\sum_{|k| \leqslant n-1} a_{k} t^{k}\right| d m(t) \\
& =\min _{a_{k} \in \mathbb{C}} \frac{1}{\rho^{n-1}} \int_{\mathbb{T}}\left|\frac{\rho^{n-1} t^{n-1}}{1-\rho t}-\sum_{k=0}^{2(n-1)} \rho^{n-1} a_{k-n+1} t^{k}\right| d m(t) \\
& =\frac{1}{\rho^{n-1}} \min _{a_{k} \in \mathbb{C}} \int_{\mathbb{T}}\left|\frac{1}{1-\rho t}-\sum_{k=0}^{n-2} \rho^{k} t^{k}-\sum_{k=0}^{2(n-1)} \rho^{n-1} a_{k-n+1} t^{k}\right| d m(t) \\
& =\frac{1}{\rho^{n-1}} \min _{b_{k} \in \mathbb{C}} \int_{\mathbb{T}}\left|\frac{1}{1-\rho t}-\sum_{k=0}^{2(n-1)} b_{k} t^{k}\right| d m(t) \\
& =\frac{1}{\rho^{n-1}} E_{2 n-1}(f)_{1} \text {. }
\end{aligned}
$$

Therefore by (1.6) we get the equality (1.5).

\section{Acknowledgment}

This research was supported by the Kyrgyz-Turkish Manas University (Bishkek/Kyrgyz Republic), project No. KTMÜ-BAP-2018.FBE.05.

\section{References}

[1] F. G. Abdullayev, G. A. Abdullayev, V. V. Savchuk, Best approximation of holomorphic functions from Hardy space in terms of Taylor coefficient, to appear in Filomat. 3

[2] S. Y. Al'per, On the Best Mean First-degree Approximation of Analytic Functions on Circle (Russian), Dokl. Akad. Nauk S.S.S.R., 153 (1963), 503-506. 4

[3] A. A. Pekarskii, Comparison of the Best Uniform Approximations of Analytic Functions in the Disk and on Its Boundary (Russian), translated from Tr. Mat. Inst. Steklova, 255 (2006), 227-232. 1, 1, 1

[4] V. V. Savchuk, Best Approximation of Cauchy-Szegö Kernel in the Mean on Circle (Ukrainian), Ukr. Mat. Zh., 70 (2018), 708-714. 4

[5] V. V. Savchuk, S. O. Chaichenko, Addendum to a theorem of F. Wiener about sieve (Ukrainian), Praci Instytutu Matematyky NAN Ukrainy, 12 (2015), 262-272. 2

[6] V. V. Savchuk, M. V. Savchuk, S. O. Chaichenko, Approximation of Analytic Functions byde Valle Poussin sums (Ukrainian), Matematychni Studii, 34 (2010), 207-219. 3 